\title{
EXAME PREVENTIVO DO COLO DO ÚTERO: PROPOSTA DE AÇÃO EDUCATIVA PARA PROMOVER A ADESÃO DE USUSÁRIAS EM UMA ESF NO SUL DE MINAS
}

\author{
João Henrique de Morais RIBEIRO ${ }^{1}$ \\ Ana Paula Alonso REIS ${ }^{2}$ \\ Marina Pereira CORTEZ ${ }^{3}$ \\ Aline Neves de OLIVEIRA ${ }^{4}$ \\ Carla Lúcia Goulart Constant ALCOFORADO ${ }^{5}$ \\ Flávia de OLIVEIRA ${ }^{6}$
}

\begin{abstract}
${ }^{1}$ Enfermeiro. Especialista em Atenção Básica em Saúde da Família. Mestrando do Programa de Pós-graduação em Enfermagem da Universidade Federal de Alfenas, UNIFAL-MG. enf.joaoh@ gmail.com

2Enfermeira. Especialista em Saúde Pública. Mestranda do Programa de Pós-graduação em Enfermagem da UNIFAL-MG. apareis@bol.com.br

${ }^{3}$ Enfermeira. Especialista em Saúde da Família e Formação Pedagógica para Profissionais de Saúde. Mestranda do Programa de Pós-graduação em Enfermagem da UNIFAL-MG. ninacortp@ hotmail.com

${ }^{4}$ Enfermeira. Especialista em Formação Pedagógica para Profissionais de Saúde. Mestranda do Programa de Pósgraduação em Enfermagem da UNIFAL-MG. aline.neoliveira@gmail.com

${ }^{5}$ Enfermeira. Docente da Escola de Enfermagem da Universidade Federal de Minas Gerais - UFMG. Doutoranda do Programa de Pós-graduação em Enfermagem UFMG. carlalcoforado@globo.com

${ }^{6}$ Enfermeira. Tutora e Orientadora do Curso de Especialização em Atenção Básica em Saúde da Família - CEABSF UNIFAL-MG/UFMG. Mestre em Enfermagem pela UNIFAL-MG. Doutoranda do Programa de Pós-graduação em Enfermagem da Universidade Federal de Minas Gerais - UFMG. flavia_efoa@yahoo.com.br
\end{abstract}

Recebido em: 30/05/2014 - Aprovado em: 20/09/2014 - Disponibilizado em: 15/12/2014

\begin{abstract}
RESUMO
O câncer de colo de útero é a patologia maligna que mais acomete as mulheres. Nesse contexto, torna-se relevante àimplementação de atividades educativas na Estratégia Saúde da Família, com finalidade preventiva e mobilizadora,com mulheres na faixa etária preconizada para realização do exame, buscando a interação entre as mesmas e possibilitando uma mudança no estilo de pensar em saúde. Trata-se de um estudo descritivo e exploratório, que utilizou dados secundários para a coleta de dados. Os resultados do diagnóstico situacional e do planejamento estratégico apontam que os problemas da unidade de ESF em estudo estão relacionados à baixa adesão de mulheres na realização da coleta do exame preventivo. Sendo assim, foi proposto um plano de ação educativa ancorado na Pedagogia Libertadora, com a finalidade de ampliar a adesão ao exame preventivo, melhorar o acolhimento e vínculo com as usuárias, bem como fortalecer ações de prevenção e promoção da saúde. O plano de ação educativa propõe atividades lúdicas, roda de conversa, sessão de filmes entre outras ações. Espera-se que com a execução desse plano, as mulheres possam se transformar em cidadãs mais informadas, cientes da necessidade de participar de ações na unidade e também dos riscos da não adesão.
\end{abstract}

Descritores:Câncer de colo do útero; educação em saúde; Estratégia Saúde da Família; Enfermagem; Promoção da Saúde.

\begin{abstract}
Cancer of the cervix is a malignant disease that affects more women. In this context, it is relevant to the implementation of educational activities in the Family Health Strategy, with preventive purposes and mobilizing for women of childbearing age, seeking the interaction between them and allowing a change in the style of thinking about health. This is a study descriptive and exploratory, with the use of secondary data. The results of the situational analysis and strategic planning indicate that the family health program unit problems in this study is related to low adherence of women in performing the collection of preventive exam. Therefore, we propose an educational action plan anchored in Liberating Pedagogy, with the aim of expanding the membership of the preventive exam, improve care and bond with the user, as well as strengthen prevention and health promotion. The action plan proposes educational play activities, conversation wheel, session films among other actions. It is expected that with the implementation of the plan, women can become more informed citizens, aware of the need to participate in the action unit and also the risks of noncompliance.
\end{abstract}

Descriptors: Uterine Cervical Neoplasms;Health Education;Family Health Strategy; Nursing; Health Promotion. 


\section{INTRODUÇÃO}

Este estudo enfatiza a importância de propor ações educativas para fortalecer a adesão de mulheres ao exame preventivo do Câncer de Colo de Útero (CCU), uma vez que essa patologia é considerada a terceira mais incidente entre as mulheres brasileiras, com exceção do câncer de pele não melanoma (INCA, 2014).

De forma lenta, a evolução do CCU passa por diferentes fases pré-clínicas detectáveis e curáveis, além de apresentar maior potencial de prevenção e cura quando comparado aos demais tipos de câncer. Maiores taxas de incidência do CCU são observadas em países em desenvolvimento, estando associadas ao baixo índice de desenvolvimento humano, precárias condições de vida, fragilidade ou ausência de ações estratégias de promoção e prevenção da saúde, além da dificuldade de acesso a serviços públicos de saúde para o diagnóstico precoce e tratamento das lesões precursoras (SILVA, 2010; VALENTE et al., 2009).

O CCU é responsável pelo grande número de morte de mulheres em idade social e economicamente ativasdevido à baixa cobertura do exame, a inadequação na coleta e na emissão de laudos colpocitológicos e a pouca adesão das mulheres ao exame são dificuldades significativas no controle desse tipo de câncer,sendo que, ao considerar estes fatores, confere a esse tipo de neoplasia, o título de problema de saúde pública brasileiro, visto que devido o seu alto potencial de cura, há uma necessidade de incrementar a oferta e estimular as mulheres a retornarem para buscar o resultado do exame (VASCONCELOSetal. 2010; SILVA; SILVEIRA; GREGÓRIO, 2012).

Dentre os fatores de risco principais para o câncer de colo de útero estão a infecção pelo Papiloma vírus Humano (HPV), parceiros múltiplos, baixo nível socioeconômico, número aumentado de gestações, uso prolongado de contraceptivos orais, tabagismo, pacientes tratadas com imunossupressores, infecção pelo Vírus da Imunodeficiência Humana (HIV) e outras doenças sexualmente transmitidas (SILVA; SILVEIRA; GREGÓRIO, 2012).

$\mathrm{O}$ vírus do papiloma humano (HPV) está presente em mais de $90 \%$ dos casos de CCU, apresentando um importante papel no desenvolvimento da neoplasia das células cervicais. Assim, como forma de prevenção primária, o uso do preservativo durante a relação sexual é umas das formas de evitar o contágio pelo HPV e este tipo de ação deve ser encorajada em todas as faixas etárias. Como ação de prevenção secundária, e para que sejam detectadas lesões precursoras para um diagnóstico precoce, o rastreamento no Brasil é feito por meio do exame preventivo, 
popularmente conhecido como Papanicolaou (FREITAS, 2009; VALENTE et al., 2009).

$\mathrm{O}$ exame preventivo do $\mathrm{CCU}$ (Papanicolau) é um teste realizado para detectar alterações nas células do colo do útero, podendo ser chamado de esfregaço cervicovaginal e colpocitologia oncótica cervical. O nome "Papanicolaou" é uma homenagem ao patologista grego Georges Papanicolaou, criador do método no início do século passado. É considerada a estratégia principal para detecção de lesões precoces, podendo ser realizado em postos ou unidades de saúde da rede pública municipal. É um exame indolor, simples e rápido e, que pode causar um pequeno desconforto que reduz quando a mulher consegue relaxar e quando o procedimento érealizado com boa técnica, de modo delicado(BRASIL, 2011).

Mesmo sendo um serviço prestado pelas unidades de saúde do SUS, podemos encontrar diversos problemas relacionados a não adesão à coleta do Papanicolau. Dentre eles, citamos a dificuldade de acesso aos serviços; crença de ser saudável relacionada ao fato de não apresentar queixas ginecológicas; medo em relação a estar com câncer e também em relaçãoao próprio procedimento; sentimentos de embaraço, constrangimento e vergonha; desconforto físico; desconhecimento da importância e da finalidade do exame; entre outros (RODRIGUES et al., 2012; THUM et al., 2008; REIS et al., 2010).
O rastreamento para CCU, através do Papanicolaou, é oferecido às mulheres brasileiras na faixa etária de 25 a 64 anos e que iniciaram suas atividades sexuais. Essa faixa etária se justifica por abarcar a maior incidência de lesões de alto grau, passíveis de efetivo tratamento (CASARIN; PICCOLI, 2011; INCA, 2014).

Observa-se que existe um sentimento de desconforto físico e psicológico frequente entre as mulheres, refletindo nas relações estabelecidas entre usuárias e os profissionais de saúde. Estes sentimentos são ocasionados pela falta de explicação do significado do exame, pela realização de forma fria e descuidada, impossibilitando ações educativas e de autoconhecimento para mulher. Nesse contexto, é fundamental o rompimento da visão tradicional de assistência à saúde, incorporando ações na visão integral, considerando além do corpo biológico, os aspectos psicológicos, econômicos, culturais e sociais da usuária. (PRADO; PEREIRA; ASSIS, 2009; THUM et al., 2008; RODRIGUES et al, 2012). A despeito disso, os aspectos subjetivos que envolvem o cotidiano da mulher e do profissional que a assiste se perdem em meio às campanhas de prevenção, distanciando-se do discurso da integralidade. Tal comportamento dificulta a obtenção de melhores resultados na realização do exame preventivo (PAULA; MADEIRA, 2003). 
Sendo assim, é necessária maior efetividade nas práticas de saúde, nas ações de educação popular, no rastreamento de mulheres que não realizam o exame preventivo e na capacitação dos profissionais que realizam esse procedimento. Além disso, passar a enxergar a mulher como um ser complexo e considerar sua posição social ao longo da história poderão auxiliar na composição e execução de novas campanhas, novos projetos e novas condutas para mulheres que procuram as unidades de saúde para a realização de exame preventivo (GUIMARÃES et al., 2012; SILVA; SILVEIRA; GREGÓRIO, 2012).

Diante do exposto, o objetivo deste estudo é propor uma atividade educativa para mulheres cadastradas em uma unidade de Saúde da Família no sul de Minas Gerais.

\section{MÉTODOS}

Trata-se de um estudo de abordagem quantitativa, descritivo e exploratório, utilizando dados secundários. Este estudo resultoudo trabalho de conclusão do Curso de Especialização em Atenção Básica em Saúde da Família, oferecido pelo Núcleo de Educação em Saúde Coletiva da Universidade Federal de Minas Gerais (UFMG) em parceria com a Universidade Federal de Alfenas, na modalidade $\mathrm{EaD}$. Na intenção de preservar o anonimato da unidade de saúde estudada, a mesma recebeu nome fictício de ESF Esperança.

Os dados secundários empregados neste estudo foram extraídos do Sistema de Informação da Atenção Básica (SIAB) disponíveis na Internet, bem como dos relatórios PMA2 e SSA2 da ESF Esperança. No Brasil, não há consenso definido sobre a necessidade de anuência do Comitê de Ética em Pesquisa quando se utiliza dados secundários, resultando na falta de norteamento para pesquisadores que realizam seus estudos empregando tal metodologia e também, não há impedimento legal para a realização de pesquisas com utilização deste tipo de dados (SOUTO et al., 2011).

Com a finalidade de identificar os principais problemasrelacionados à baixa adesão na realização do exame de papanicolauda área de abrangência da ESF Esperança, e produzir informações sobre as causas e consequências destes problemas, foi realizado o Diagnóstico Situacional da Unidade, com base no método da Estimativa Rápida. Entende-se por Estimativa Rápida um modo para obtenção de informações sobre um conjunto de problemas e dos recursos necessários para seu enfrentamento, constituindo importante ferramenta no processo de planejamento participativo. $\mathrm{O}$ termo "rápida" refere-se ao tempo gasto entre a coleta e análise de dados, devendo este ser o mínimo necessário para angariar informações 
relevantes no campo (CAMPOS; FARIA; SANTOS, 2010).

Para delinear o problema descoberto no diagnóstico situacional, foi executado o planejamento e a elaboração do plano de atividade educativa, como estratégia assertiva para solucionar o problema da unidade de saúde, bem como atender de forma integral e acolhedora a população do território.

\section{RESULTADOS}

Por meio do diagnóstico situacional da ESF Esperança, foi verificado que os principais problemas do território estão relacionados à baixa adesão das mulheres na coleta do exame preventivo, fato que pode estar relacionado ao medo e vergonha de realizar o exame na unidade, o baixo nível educacional das mulheres cadastradas na unidade, bem como a falta de informação da importância na realização do exame preventivo.

Com um número expressivo de mulheres na faixa etária proposta para a realização do exame preventivo para Câncer de Colo do Útero (6880 mulheres entre 25 e 64 anos) e comparado ao número de exames realizados no município (84 exames realizados em novembro de 2012) e na unidade (em média três exames mensais), depara-se com percentual abaixo da meta preconizada pelo Programa Viva Mulher neste período, sendo que a mesma estabelecia a realização de 182 exames preventivos, atingindo assim, 46,15\% do estipulado. Preconizado (SISCOLO, 2014).

Os problemas da população da unidade de ESF Esperançaforam discutidos entre os pesquisadores e Equipe de Saúde, com vistas a priorizar as ações para resolução dos problemas. Após discussão, foi eleita como prioridade a baixa adesão de mulheres ao exame preventivo e para tanto, foram elencados os nós críticos que poderiam estar interferindo nesta adesão, tais como:

$\checkmark$ Falta de informação das mulheres quanto à importância da realização do exame preventivo para câncer de colo do útero;

$\checkmark$ Baixo nível socioeconômico da população;

$\checkmark$ Medo do procedimento;

$\checkmark$ Preconceito e vergonha por parte das mulheres;

$\checkmark$ Fragilidade no vínculo.

A Figura 01 delimita os nós críticos elencados e proporciona a incorporação desses nós às ações estratégicas da equipe, visando ampliação da adesão das mulheres na realização do exame preventivo, bem como a participação destas em ações educativas na unidade.

Diante da complexidade do problema, foi indicada a criação de um grupo operativo, por meio de uma ação educativa estruturada,na tentativa de superar todas as faces desteproblema.Nesse contexto, é de 
extrema importância a identificação dos atores que controlam os recursos necessários para a realização de tal grupo, analisando seu posicionamento e motivação para que então, possam ser definidas ações e estratégias de solução para os nós críticos identificados.

A motivação pode ser classificada em favorável quando o ator que controla o recurso crítico transfere o controle do recurso para a equipe de planejamento; indiferente, quando não há clareza e garantia da utilização desse recurso; e por fim, contrária, considerada a oposição ativa quanto ao plano de ação (CAMPOS; FARIA; SANTOS, 2010).

O plano proposto apresenta recursos críticos de ordem política, organizacional e financeira e, a motivação dos atores que controlam tais recursos é, em sua grande maioria, favorável.

Figura 01: Atividades da equipe de ESF, problema identificado e estratégia de resolução. ESF Esperança, 2012.

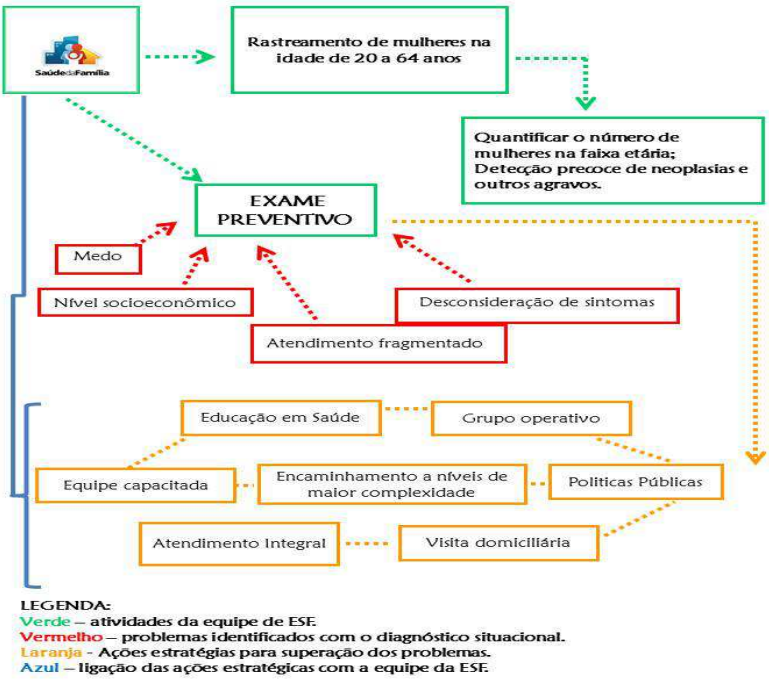

Fonte: RIBEIRO, J.H. M. et al. 2014.

\section{O plano e atividade educativa- PAE}

Educação e saúde são espaços na qual ocorrem interseção entre os níveis de atenção a saúde e a obtenção contínua de conhecimentos pelos profissionais de saúde, além de produzir e aplicar os saberes destinados ao desenvolvimento humano. Dessa forma, há um consecutivo ciclo de ensinar e aprender entre estes profissionais, muitas vezes, inconscientemente (PEREIRA, 2003). Nesse contexto, o educar não é uma simples transmissão de conhecimentos, pois está amparado em um arcabouço de representações sociais e de homem que se deseja formar; é por meio da educação, que códigos sociais e valores culturais são reproduzidos e transformados (LUCKESI, 1994; SAVIANE, 1985).

Sendo assim, optamos por elaborar um PAEancorado nos ideais da pedagogia libertadora ou da problematização, por esta permitir uma prática educativa mais participativa, podendo ser direcionada tanto à população quanto aos profissionais de saúde. A adoção de tal pedagogia estádiretamente relacionada à prática da Promoção da Saúde, quando $\mathrm{o}$ incremento do poder (empowerment) pessoal e comunitário favorece o desenvolvimento de atitudes e habilidades para atuar em prol de sua saúde, indo de encontro com as ações propostas pela Carta de Ottawa. Esse empowerment está relacionado à criação de programas 
educativos que favoreçam a formação de uma consciência crítica sobre a realidade vivida (BRASIL, 2002; LAVERACK, 2001).

Portanto, o processo de aprendizagem neste tipo de ação educativa ocorre por meio de grupos de discussão, na busca por uma relação dialógica entre os atores da aprendizagem. Assim, podemos construir conhecimento sobre uma realidade concreta, vivenciada pelos usuários e não por imposição ou memorização de temas. Dessa forma, o aprendizado será por meio da compreensão e reflexão da realidade e não pela transmissão de conhecimentos proposta por outros métodos pedagógicos (LIBÂNEO, 1983).

O plano recebeu o nome de TPM Tornando o Preventivo Maravilhoso e é composto das seguintes ações:

Ementa: Câncer no Brasil; Tipos frequentes de câncer que afetam as mulheres; câncer de colo do útero; câncer de mama, exame preventivo; acolhimento; rastreamento; diagnóstico e tratamento; sintomas importantes; cuidados necessários.

Público-alvo: mulheres cadastradas na USF Esperança, com idade entre 25 e 64 anos.

Metodologia: exposições orais; rodas de conversa; teatros e dramatizações; sessão filme; oficinas lúdicas.
Exposições orais: serão realizadas pelos profissionais da unidade: médico, enfermeiro e dentista.

Rodas de conversa: a proposta é incentivar as mulheres a expor suas expectativas, medos e angústias em relação ao exame preventivo, tendo como moderador, o enfermeiro da unidade.

Teatros e dramatizações: utilizando a técnica de role playing, as mulheres serão convidadas a dramatizar as situações vivenciadas e propostas pela equipe, com finalidade de sensibilizar as integrantes. Nesse espaço, contaremos com os Agentes Comunitários de Saúde, enfermeiro e auxiliar de enfermagem.

Sessão filme: serão exibidos filmes que envolvem a temática proposta pelo grupo para subsidiar a discussão nas rodas de conversa..

Oficinas lúdicas: com caráter informativo, as oficinas visam aumentar o nível de instrução das mulheres quanto à necessidade de adesão ao exame preventivo.

Finalmente, o PAE foi elaborado na tentativa de maximizar ações de promoção e prevenção na ESF Esperança, bem como favorecer o vínculo entre usuários e profissionais de saúde, permitindo maior adesão das usuárias na realização do exame Papanicolaou. 


\section{CONSIDERAÇÕES FINAIS}

Diante do grande número de novos casos de câncer de colo do útero, torna-se imperativo a adesão das mulheres quanto à realização do exame preventivo Papanicolaou. Neste estudo, fica evidente a necessidade de propor alternativas para que as usuárias da ESF passem a aderir à realização deste exame, bem como ações de promoção e prevenção, visando à manutenção da saúde e qualidade de vida.

Espera-se que o plano de atividade educativa consiga transformar essas mulheres em cidadãs mais informadas, cientes da necessidade de participar de ações na unidade e também dos riscos da não adesão. Não era nosso objetivo avaliar o plano de ação, mas sim, propor uma estratégia eficaz e eficiente para superação do problema da população feminina da ESF em estudo.

Por fim, aponta-se como limitação para a realização deste estudo, a dificuldade de acesso aos dados do município no sistema de informação online, bem como a falta de interesse de profissionais e gestores da ESF estudada, no período de coleta de dados.

\section{REFERENCIAS}

BRASIL, Ministério da Saúde. Exame preventivo do câncer de colo uterino (Papanicolau). Série Dicas em Saúde. Elaborada em julho de 2011. Disponível em: http://bvsms.saude.gov.br/bvs/dicas/237_papa nicolau.html. Acesso em: 24 nov. 2013.

BRASIL. Ministério da Saúde. Política Nacional de Promoção da Saúde: documento para discussão. Brasília, DF: Editora MS; 2002.

CAMPOS, F.C.C.; FARIA, H.P.; SANTOS, M.A. Planejamento e avaliação das ações em saúde. $2^{\mathrm{a}}$ ed. Belo Horizonte: Nescon/UFMG, 2010.

CASARIN, M.R.; PICOLLI, J.C.E. Educação em saúde para prevenção do câncer de colo do útero em mulheres do município de Santo Ângelo/RS. Ciênc. saúde coletiva. 16(9):3925-3932. 2011.

FREITAS,G. L, et al. Discutindo a política de atenção à saúde da mulher no contexto da promoção da saúde. Rev. Eletr. Enf.11(2):424-428.2009. Disponível em: http://www.fen.ufg.br/fen_revista/v11/n2/v11 n2a26.htm. Acesso em: 19 nov. 2013.

GUIMARÃES, J.A.F et al. Pesquisa brasileira sobre prevenção do câncer de colo uterino: uma revisão integrativa. Rev Rene. 13(1):220-30.2012. Disponível em: http://www.revistarene.ufc.br/revista/index.ph p/revista/article/view/34/29. Acesso em: 19 nov. 2013.

INSTITUTO NACIONAL DO CÂNCER. Controle do Câncer de Colo de Útero. 
Disponível

em:

http://www2.inca.gov.br/wps/wcm/connect/ac oes_programas/site/home/nobrasil/programa nacional_controle_cancer_colo_utero. Acesso em: 22 ago. 2014.

INSTITUTO NACIONAL DO CÂNCER. Controle do Câncer de Colo de Útero: Detecção Precoce. Disponível em: http://www2.inca.gov.br/wps/wcm/connect/ac oes_programas/site/home/nobrasil/programa nacional_controle_cancer_colo_utero/detecca o_precoce. Acesso em: 22 ago. 2014.

LAVERACK, G. An identification and interpretation of the organizational aspects of community empowerment.CommunityDev J.36(2):134-146.2001.

LIBÂNEO, J. C.. Tendenciaspedagogicas em lapráctica escolar. Revista da Associação Nacional de Educação $\neg$ ANDE. 1983. Disponível em: http://www.fba.unlp.edu.ar/musica/fundament ostem/wpcontent/uploads/2012/05/U5_Texto-n5_2013.pdf. Acesso em: 16 nov. 2013.

LUCKESI, C. C.. Filosofia da Educação. São Paulo: Cortez Editora,1994.

PAULA, A. F.; MADEIRA, A. O exame colpocitológico sob a ótica da mulher que o vivencia. . Rev. esc. enferm. USP. 37(3):8896.2003 .
PEREIRA, A.L.F. As tendências pedagógicas e a prática educativa nas ciências da saúde. Cad. Saúde Pública. 19(5):1527-1534. 2003.

PRADO, E.V.; PEREIRA, W.S.B.; ASSIS, M. Reorganização das ações de prevenção do câncer ginecológico a partir da educação popular em saúde: a experiência da equipe urbana da estratégia de saúde da família de Rio Negro/MS. Rev. APS. 12(4):498-503. 2009.

REIS, A.A.S. et al. Papilomavírus humano e saúde pública: prevenção ao carcinoma de cérvice uterina. Ciênc. saúde coletiva. 15:S1055-S1060. 2010. Suplemento 1.

RODRIGUES, B.C. et al. Educação em saúde para a prevenção do câncer cérvico-uterino. Rev. bras. educ. med. 36(1):S149-S154. 2012. Suplemento 1.

SAVIANE, D.. Escola e Democracia. 6a Ed. São Paulo: Cortez Editora, 1985.

SILVA, A.P.S. Efeitos da aplicabilidade de um manual educativo para ações de detecção precoce do câncer de mama. 2012. 138f. Tese (Doutorado. Faculdade de Farmácia, Odontologia e Enfermagem, Universidade Federal do Ceará, Fortaleza, 2012. Acesso em: http://www.repositorio.ufc.br/ri/bitstream/riuf 
c/6998/1/2012_tese_apssilva.pdf. Acesso em: 30 nov. 2013.

SILVA, S.R.; SILVEIRA, C.F.; GREGÓRIO, C.C.M. Motivos alegados para a não realização do exame de papanicolaou, segundo mulheres em tratamento quimioterápico contra o câncer do colo uterino. Rev. min. enferm. 16(4):579-587. 2012. Disponível em: http://pesquisa.bvs.br/brasil/resource/pt/bde23941.Acesso em: 15 nov. 2013.

SISCOLO. Programa Nacional de Controle do Câncer do colo do útero e mama de Minas Gerais. Disponível em: http://mg.vivamulher.com.br/. Acesso em: 31 ago. 2014.

SOUTO, R. Q. et al. Aspectos éticos na pesquisa com dados secundários: uma revisão sistemática. Revista Espaço para a Saúde. 13(1):45-53. 2011. Disponível em: http://www.uel.br/revistas/uel/index.php/espa coparasaude/article/view/9728/pdf. Acesso em: 09 nov. 2013.

THUM, M. et al. Câncer de colo uterino: percepção das mulheres sobre prevenção. CiencCuidSaude. 7(4):509-516. 2008.

Disponível em: http://www.periodicos.uem.br/ojs/index.php/ CiencCuidSaude/article/view/6659/3917. Acesso em 18 nov. 2013.
VALENTE, C.A. et al. Conhecimento de mulheres sobre o exame de papanicolaou. Rev. esc. enferm. USP. 43(2):1193-1198. 2009.

VASCONCELOS, C.T. M. et al. Análise da cobertura e dos exames colpocitológicos não retirados de uma Unidade Básica de Saúde. Rev. esc. enferm. USP. 44(2):324-330. 2010. : 